\title{
Reducing Emergency Department Visits and Opioid-Related Deaths in Maryland
}

\author{
$\underline{\text { Spencer Andrews }}^{1^{*}}, \underline{\text { Cara M. DeAngelis }}^{2 *}, \underline{\text { Somayeh }}$ \\ Hooshmand $^{3 *}$, Neysha Martinez-Orengo ${ }^{4 *}$, Melissa Zajdel ${ }^{5 *}$ \\ 1'Indiana University, Bloomington, IN \\ 2 University of Toledo College of Medicine and Life Sciences, Toledo, $\mathrm{OH}$ \\ ${ }^{3}$ Old Dominion University, Norfolk, VA \\ 4Ponce Health Sciences University, Ponce, PR \\ ${ }^{5}$ Carnegie Mellon University, Pittsburgh, PA \\ *All authors contributed equally \\ https://doi.org/10.38126/JSPG180403 \\ Corresponding author: neyshamo@gmail.com \\ Keywords: opioid epidemic; Maryland HOPE and Treatment Act of 2017; hospital discharge; opioid use \\ disorder; peer recovery specialist; vulnerable populations; Maryland General Assembly
}

\begin{abstract}
Executive Summary: The state of Maryland has consistently ranked among the top states by opioid-involved overdose deaths. Emergency rooms in Maryland have been overrun with patients struggling with opioid use disorder (OUD). While hospitals are heavily burdened, it has become clear that they serve as a critical entry point for OUD prevention programs. Despite this, when section 19-310 of the Maryland Heroin and Opioid Prevention Effort (HOPE) and Treatment Act of 2017 passed, it included vague language requiring hospitals to create their own discharge protocols for such patients rather than putting into place statewide mandates. We propose two alternative solutions. First, the Maryland General Assembly can amend the HOPE and Treatment Act of 2017 to mandate that peer recovery services be made available during inpatient care, within the emergency department, and post-discharge for patients presenting with OUD. Second, we recommend the addition of a subtitle to describe how to establish and operate mobile clinic treatment programs. The former amendment would offer a prompt solution that could reduce opioid-related hospitalizations and deaths in the state. It will also help reach underrepresented populations who are the least likely to access peer recovery support and other health services in response to OUD.
\end{abstract}

I. The cost of the opioid epidemic in Maryland and its burden on emergency departments

According to the most recent estimates, Maryland has the second highest opioid-related death rate in the United States (NIDA 2020). In fact, last year the state reported the largest annual total opioid-related deaths on record (OOCC 2020). While causing widespread devastation, the ongoing opioid epidemic has particularly burdened hospital emergency departments (EDs). Recently, the national costs associated with emergency room visits due to opioidrelated issues reached nearly $\$ 5$ billion per year (Langabeer et al. 2021). In 2014, Maryland had the highest opioid-related hospitalization rates nationally (Weiss et al. 2017). Since then, the Maryland opioid-related hospitalization rate has outpaced the national average every year (HCUP 2021). Furthermore, as seen with COVID-19, health crises can co-occur, exacerbating the strain on healthcare systems. For example, ED visits for opioid overdoses significantly increased nationwide in March to October in 2020 compared to the same period in 2019 (Holland 2021).

The frequent and sometimes recurrent use of hospital services by individuals with opioid use disorder (OUD) makes EDs a crucial access point for intervention with referral to treatment centers and 
peer recovery support (Hawk et al. 2019). However, there is substantial variability in treatment adherence after hospital discharge. After discharge from an initial hospital visit due to overdose, Black patients are half as likely to obtain follow-up appointments for OUD care compared to White patients; women, Hispanic patients, and older individuals are also less likely to complete OUD treatment after hospital discharge (Kilaru et al. 2020).

One approach to decrease opioid-related rehospitalization and death rates is by connecting patients with peer recovery support specialists. Peer recovery specialists have lived experience with substance use and can help patients connect with community resources and medication-assisted treatment. Such groups have shown to be particularly valuable in engaging with racial and ethnic minority groups who have limited access to healthcare due to logistical barriers, marginalization, and stigma (van Draanen et al. 2020). Recent ED discharge programs, which have included peer recovery support in states including Indiana, New Jersey, and Nevada, have shown effective preliminary outcomes, including reduced rates of opioid use and hospitalizations (McGuire et al. 2019).

\section{Opioid epidemic as an intersectional crisis}

The societal costs of the opioid epidemic are as significant as the economic costs; comprehensive discussion about the opioid crisis must examine the problem and solutions through an intersectional lens. Although rates of death due to opioid overdoses in the U.S. decreased overall between 2017 and 2018, rates increased for both Black and Hispanic populations (Wilson et al. 2020). Additionally, overdose rates have increased more sharply for Black Americans (44\%) than White Americans (22\%) (James and Jordan 2018). Low socioeconomic status also influences vulnerability and correlates with higher risk of developing a substance use disorder among already at-risk populations, e.g., individuals with mental health conditions (Dasgupta et al. 2018).

The COVID-19 pandemic has exacerbated preexisting health disparities. Black Americans are not only more likely than White Americans to contract COVID-19, but Black patients with COVID-19 and substance use disorders, especially individuals with OUD, are more likely to die due to COVID-19 when compared to their White counterparts. This is consistent with data that suggests Black individuals are more likely to suffer adverse outcomes from COVID-19 (Wang et al. 2020). However, there are tools popularized during the pandemic - such as telemedicine and mobile health clinics-that may facilitate access to healthcare in these vulnerable populations and address health disparities. For example, a mobile clinic offering medication for OUD outside the Baltimore City jail resulted in 32\% retention after thirty days of initial treatment. Patients (primarily African American) did not only return for additional visits, but of those who initiated care, $22 \%$ transferred to continue buprenorphine treatment-one of three common medications used to treat OUD-at a partner site (Krawczyk et al. 2019). Other studies looking at databases of patients receiving buprenorphine medication-assisted treatment reported approximately 39\% adherence in a twelve-month period (Ronquest et al. 2018). Buprenorphine is typically preferred over other medication options due to its reduced addiction potential; however, buprenorphine is primarily available to White individuals with OUD, while the other options are more readily accessible to Black and Hispanic communities (Goedel et al. 2020). This shows the potential of mobile clinics for successfully reaching vulnerable populations and creating connections with patients in order to support treatment adherence.

\section{Maryland's response}

Responding to the epidemic, Maryland legislators passed the Heroin and Opioid Prevention Effort (HOPE) and Treatment Act in June 2017, laying out the state's plan to control its growing opioid crisis. Under this Act, hospitals are required to have a protocol in place for discharging and assisting patients treated for OUD. However, vague language in the bill left hospitals in Maryland to submit individual discharge protocols instead of ensuring that patient care and discharge protocols across all Maryland hospitals included connecting with peer recovery specialists support (Stallings and Braithwaite 2018).

In order to have a comprehensive and coordinated response to the opioid crisis, Maryland has established Opioid Intervention Teams (OITs) in each of its twenty-four counties. These teams are multiagency coordinating bodies that consult and report on the implementation of specific high-priority 
programs and services to the Opioid Operational Command Center (OOCC). The OIT program inventory list includes having peer recovery specialists at the hospitals and at other sites intended to support treatment adherence upon discharge. Despite OITs recommending the implementation of these services, counties with the largest percent increases in opioid-related deaths still lack plans for implementing peer-recovery specialists (e.g., Allegany and Charles). Moreover, only eight out of twenty-four counties have implemented peer recovery specialist support in $\geq 80 \%$ (at least eight out of ten services) of OIT recommended programming (Figure 1).

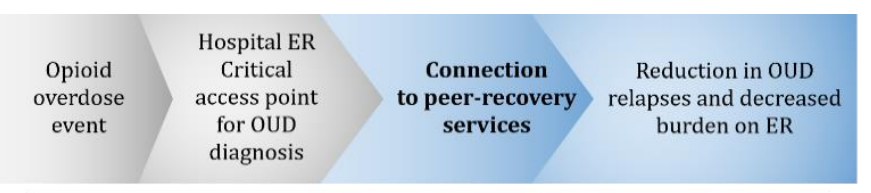

Implementation of certified peer-recovery specialist by county

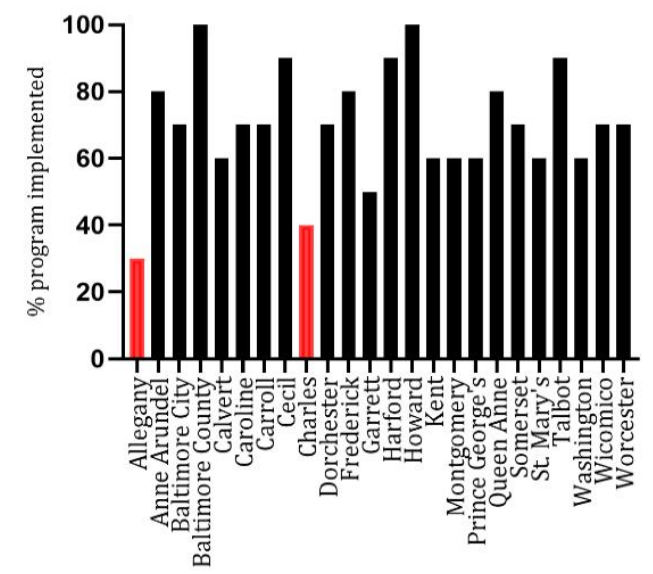

Figure 1: Implementation of peer recovery specialists in Maryland. Top schematic depicts the role of hospitals as a crucial entry point for peer support intervention for patients with OUD. Bottom graph shows the percentage of peer recovery specialist programming implemented by county. Counties in red not only have the lowest percentage of implemented peer recovery specialists, but they also have the largest percent increase of opioidrelated deaths during the years 2019-2020. Figure created using data from the Maryland Opioid Operational Command Center 2020 Annual Report (OOCC 2020).

Another observation is that the Act does not include the implementation and operation of mobile clinics, despite their ability to reach vulnerable communities, expand preventive services, and tackle limitations of the traditional health care system (Malone et al. 2020). Although the OITs also incorporate RV/Truckbased treatment as part of their program inventory, unfortunately only $17 \%$ of the counties have fully implemented this program.

The previous data show that there is still a disconnect between what the Maryland HOPE and Treatment Act of 2017 mandates and what is needed to help reduce ED visits and opioid-related deaths. In this memo, we explore two policy options that describe amendments to the Act. Both options aim to advance care planning after hospital discharge and improve community access to OUD treatment and peer recovery specialists.

\section{Policy options}

Two amendments to the Maryland HOPE and Treatment Act of 2017 are proposed to help reduce ED visits and opioid-related deaths in Maryland.

\section{i. Option 1: Mandate peer recovery specialist support for opioid use disorder}

Section 19-310 of the HOPE and Treatment Act of 2017 requires that hospitals "have protocols for discharging patients treated for a drug overdose or identified as having a substance use disorder." The bill states that these discharge protocols "may" include peer recovery specialists who can make referral to treatment or connect the patient with community services. It mandates no specific requirements, and as a result, each hospital is left to create their own protocols. In this way, the bill fails to adequately support individuals with OUD, who are often uniquely dependent on long-term interventions for treatment. Without mandating that hospitals connect such patients to peer-support interventions, individuals may be more likely to continue opioid use, continue to burden hospital emergency rooms, and increase the risk of death.

We propose that this statute be amended to eliminate non-specific language in section 19-310 and mandate that the discharge protocols for all Maryland hospitals for OUD and opioid overdose patients include connecting such patients to peer recovery specialist services. These services should be made available in the ED, inpatient settings, and most importantly, after discharge while back in the community.

\section{Advantages}

Since peer recovery support has shown to be an effective facilitator of recovery, this proposed 
amendment could reduce emergency room overflow by slowing the cyclical return of some individuals suffering from OUD and thereby alleviate the burden on hospital EDs. Additionally, minority and vulnerable populations are less likely to return for follow-up visits, and including peer recovery services for all patients may reduce these disparities. Mandating that hospitals provide peer recovery services and connect patients with such services after discharge will help to facilitate continuity of care, which can be incredibly important for effective continued participation in treatment.

\section{Disadvantages}

This amendment could improve outcomes for individuals with OUD, but not all counties in Maryland have robust access to the resources required to implement peer recovery services or organized community-based treatment centers. To avoid discrepancies in care, some hospitals may need to seek additional funding, such as that provided by the Substance Abuse and Mental Health Services Administration's Opioid State Targeted Response Grants. Likewise, while it is the hospital's job to connect patients to such resources during discharge, some individuals, specifically those from underrepresented communities who tend to be less trusting of the medical establishment, may decline participation in such efforts. Additional resources and community outreach may be needed to educate, reduce mistrust of groups feeling they are being used for research, increase diversity in the healthcare field, and reduce prejudice-some of the reasons why minority groups do not trust the healthcare system. A final important point to consider is that peer-support services are not covered by the various Medicaid authorities in the state of Maryland. While the topic of broad Medicaid coverage for discharge services is not within the scope of this policy memo, further consideration should be given in the future to make these services as affordable as possible (MACPAC 2019).

ii. Option 2: Add a subtitle in the Act describing the establishment and operation of mobile clinic treatment programs

One way to directly reach underserved communities struggling with OUD is through the implementation of mobile clinics. The Maryland Opioid Operational Command Center recognizes the importance of mobile treatment services and included them among local best practices in their 2020 Report (OOCC 2020). However, most counties have not implemented RV/Truck-based treatment programs. One of the reasons for this limited performance could be the ambiguous classification of mobile clinics within the healthcare system and not providing guidelines for the implementation of these services. In the Maryland HOPE and Treatment Act of 2017, subtitle 5 describes how to establish and operate a health crisis hotline. Similarly, we propose that this statute be amended to include an additional subtitle describing how to establish and operate mobile clinics to effectively connect individuals with OUD to treatment.

\section{Advantages}

In the interest of the government's responsibility to provide quality care for vulnerable populations, the proposed amendment would establish and support mobile clinics as a healthcare facility. With approximately 2,000 mobile clinics in the US providing care to seven million people per year, these have proven to be an effective healthcare system model that compensates for gaps (e.g., closed hospitals, low reimbursement from insurers, and physician and other staff shortages) in traditional healthcare (Morgan 2021). Mobile clinics have the advantage of going exactly to the communities in greatest need and at the time they need it, reducing barriers to access while efficiently and flexibly distributing resources to meet demand. Because they offer preventive service, mobile clinics can also save a significant amount of money to the healthcare system (Malone et al. 2020). The incorporation of mobile clinics would not be foreign to Maryland; in February 2019, Lt. Governor Rutherford announced the Maryland Mobile Wellness Initiative to offer counseling, blood tests, naloxone, and resources to help individuals connect to long-term treatment in Annapolis.

\section{Disadvantages}

Strong connections between hospitals, pharmacies, laboratories, and other programs are needed for wellcoordinated service and continuity of care. A considerable amount of time may be needed to achieve coordination between facilities, comply with any state regulation and government certifications, and hire the required healthcare professionals to effectively manage mobile clinics. Startup costs to develop mobile clinics also represent a financial 
challenge, and maintenance may lack dedicated funding, even with the improved access to resources facilitated by the proposed change. Finally, patient privacy may be difficult to maintain in mobile settings due to limited space, which may dissuade certain patients from seeking help through these outlets.

\section{iii. Consequences of inaction}

Opioid fatalities in Maryland have continued to increase, and the state has among the highest opioidinvolved overdose hospitalization (Weiss et al. 2017) and death rates (NIDA 2020). Without either of the interventions above, the state's healthcare system will likely continue to be muddled with patientsmany returning in crisis due to opioid use. Hit hard by the COVID-19 pandemic, Maryland hospitals struggled, at points, to handle the influx of new patients in need of immediate help. This poignantly underscores the importance of hospital emergency rooms reserving capacity for individuals experiencing a less preventable health crisis.

\section{Policy recommendation}

We recommend that the Maryland State Legislature enact Policy Option 1 and amend section 19-310 of the Maryland HOPE and Treatment Act of 2017 to mandate that each Maryland hospital offer and connect individuals with OUD with peer recovery support services. This amendment would provide a statewide, long-term solution for individuals with OUD admitted to hospitals-a critical entry point for

\section{References}

Ashford, Robert D., Matthew Meeks, Brenda Curtis, and Austin M. Brown. "Utilization of peer-based substance use disorder and recovery interventions in rural emergency departments: Patient characteristics and exploratory analysis." (2019). Journal of Rural Mental Health 43(1): 1729. https://doi.org/10.1037/rmh0000106.

Dasgupta, Nabarun, Leo Beletsky, and Daniel Ciccarone. 2018. "Opioid Crisis: No Easy Fix to Its Social and Economic Determinants." American Journal of Public Health 108 (2): 182-86. https://doi.org/10.2105/ajph.2017.304187.

Goedel, William C., Aaron Shapiro, Magdalena Cerdá, Jennifer W. Tsai, Scott E. Hadland, and Brandon DL Marshall. 2020. "Association of racial/ethnic segregation with treatment capacity for opioid use disorder in counties in the United States." JAMA Network Open 3(4): e203711-e203711. https://doi.org/10.1001/jamanetworkopen.2020 .3711. substance use intervention. Additionally, making this change could especially help underrepresented populations, who are the least likely to access peer or professional help in response to OUD. Indeed, "warm handoff" programs-wherein patients are directly connected with a recovery program-have been linked to increased engagement in treatment programs in underserved areas (Ashford et al. 2019).

The current opioid epidemic in the U.S. continues to plague the state of Maryland and requires immediate action by the state. To reduce its burden on hospital emergency rooms, two proposed policy options offer alternative mitigating measures. Mandating the incorporation of peer recovery specialists to all hospital discharge protocols is a long-term change that may reduce rehospitalizations across the entire state of Maryland. However, since this is such a pressing issue, further action may be needed to address the needs of individuals with OUD in specific urban and rural centers where opioid use is particularly widespread. In this case, mobile clinics could serve as a nearer-term solution by offering immediate alternative care centers to reduce the burden on emergency rooms. While we believe the state would benefit from both suggested policies, the mandate that ED discharge protocols connect patients to peer recovery services should be prioritized, as it makes both immediate and lasting change to most directly address the state's ongoing crisis.

Hawk, Kathryn F., Rachel L. Glick, Arthur R. Jey, Sydney Gaylor, Jamie Doucet, Michael P. Wilson, and John S. Rozel. 2019. "Emergency Medicine Research Priorities for Early Intervention for Substance Use Disorders." The Western Journal of Emergency Medicine. Department of Emergency Medicine, University of California, Irvine School of Medicine. https://www.ncbi.nlm.nih.gov/pmc/articles/PM C6404722/.

HCUP NIS Database Documentation. Healthcare Cost and Utilization Project (HCUP). April 2021. Agency for Healthcare Research and Quality, Rockville, MD. https://www.hcupus.ahrq.gov/db/nation/nis/nisdbdocumentation. isp.

Heroin and Opioid Prevention Effort (HOPE) and Treatment Act of 2017. Maryland General Assembly. Accessed April 15, 2021. https://mgaleg.maryland.gov/mgawebsite/legisl ation/details/sb0967?ys=2017rs. 
Holland, Kristin M. 2021. "Mental Health, Overdose, and Violence Outcomes and the COVID-19 Pandemic." JAMA Psychiatry. JAMA Network. April 1. https://jamanetwork.com/journals/iamapsychia try/fullarticle/2775991.

James, Keturah, and Ayana Jordan. 2018. "The Opioid Crisis in Black Communities." Journal of Law, Medicine \& Ethics 46 (2): 404-21.

https://doi.org/10.1177/1073110518782949.

Kilaru, Austin S., Aria Xiong, Margaret Lowenstein, Zachary F. Meisel, Jeanmarie Perrone, Utsha Khatri, et al. 2020. "Incidence of Treatment for Opioid Use Disorder Following Nonfatal Overdose in Commercially Insured Patients." JAMA Network Open 3 (5).

https://doi.org/10.1001/jamanetworkopen.2020 .5852 .

Krawczyk, Noa, Megan Buresh, Michael S. Gordon, Thomas R. Blue, Michael I. Fingerhood, and Deborah Agus. 2019. "Expanding Low-Threshold Buprenorphine to Justice-Involved Individuals through Mobile Treatment: Addressing a Critical Care Gap." Journal of Substance Abuse Treatment 103: 1-8. https://doi.org/10.1016/i.jsat.2019.05.002.

Langabeer, James R., Angela L. Stotts, Bentley J. Bobrow, Henry E. Wang, Kimberly A. Chambers, Andrea J. Yatsco, et al. 2021. "Prevalence and Charges of Opioid-Related Visits to U.S. Emergency Departments." Drug and Alcohol Dependence. Elsevier. February 3.

https://www.sciencedirect.com/science/article/ abs/pii/S0376871621000636.

Malone, Nelson C., Mollie M. Williams, Mary C. Smith Fawzi, Jennifer Bennet, Caterina Hill, Jeffrey N. Katz \& Nancy E. Oriol, et al. 2020. "Mobile health clinics in the United States". International Journal for Equity in Health.

https://doi.org/10.1186/s12939-020-1135-7.

Medicaid and CHIP Payment and Access Commission (MACPAC). 2019. "Recovery Support Services for Medicaid Beneficiaries with a Substance Use Disorder." Medicaid and CHIP Payment and Access Commission.

https://www.macpac.gov/wpcontent/uploads/2019/07/Recovery-SupportServices-for-Medicaid-Beneficiaries-with-aSubstance-Use-Disorder.pdf.

McGuire, Alan B., Kristen Gilmore Powell, Peter C. Treitler, Karla D. Wagner, Krysti P. Smith, Nina Cooperman, et al. 2020. "Emergency Department-Based Peer Support for Opioid Use Disorder: Emergent Functions and Forms." Journal of Substance Abuse Treatment 108: 82-87. https://doi.org/10.1016/i.jsat.2019.06.013.
Morgan Larissa. 2021. "Highway to Better Health Care." Accessed July 20, 2021.

https://www.theregreview.org/2021/02/09/mo rgan-highway-better-health-care/.

National Institute on Drug Abuse (NIDA). 2020. "Opioid Summaries by State." National Institute on Drug Abuse.

https://www.drugabuse.gov/drugtopics/opioids/opioid-summaries-by-state.

Opioid Operational Command Center (OOCC). Annual Report January 1, 2020-December 31, 2020. Accessed July 27, 2021.

https://beforeitstoolate.maryland.gov/wpcontent/uploads/sites/34/2021/04/2020Annual-Report-Final.pdf.

Ronquest, Naoko A., Tina M Willson, Leslie B Montejano, Vijay R Nadipelli, and Bernd A. Wollschlaeger. 2018. "Relationship between buprenorphine adherence and relapse, health care utilization and costs in privately and publicly insured patients with opioid use disorder." Substance Abuse and Rehabilitation 9: 59-78. https://doi.org/10.2147/SAR.S150253.

Stallings, Nicole, and Shamonda Braithwaite. 2018. "Emergency Discharge Protocols for Patients with Substance Use Disorders and Opioid Overdoses in Maryland's Hospitals."

Maryland Hospital Association.

Van Draanen, Jenna, Christie Tsang, Sanjana Mitra, Mohammad Karamouzian, and Lindsey Richardson. 2020.2 "Socioeconomic Marginalization and Opioid-Related Overdose: A Systematic Review." Drug and Alcohol Dependence. Elsevier. June 20.

https://www.sciencedirect.com/science/article/ abs/pii/S0376871620302921.

Wang, Quan Qiu, David C. Kaelber, Rong Xu, and Nora D. Volkow. 2020. "COVID-19 Risk and Outcomes in Patients with Substance Use Disorders: Analyses from Electronic Health Records in the United States." Molecular Psychiatry 26 (1): 30-39. https://doi.org/10.1038/s41380-020-00880-7.

Weiss, Audrey J., Anne Elixhauser, Marguerite L. Barrett, Claudia A. Steiner, Molly K. Bailey, and Lauren O'Malley. 2017. “Opioid-Related Inpatient Stays and Emergency Department Visits by State, 20092014." Healthcare Cost and Utilization Project (HCUP) Statistical Briefs [Internet]. U.S. National Library of Medicine. Accessed April 30. https://www.ncbi.nlm.nih.gov/books/NBK44164 8/.

Wilson, Nana, Mbabazi Kariisa, Puja Seth, Herschel Smith, and Nicole L. Davis. 2020. "Drug and OpioidInvolved Overdose Deaths — United States, 20172018." MMWR. Morbidity and Mortality Weekly Report 69 (11): 290-97. https://doi.org/10.15585/mmwr.mm6911a4. 
Spencer Andrews is currently a postbaccalaureate fellow studying object and face recognition pathways in the brain. He holds a B.S. in Neuroscience from Indiana University in Bloomington, Indiana.

Cara DeAngelis is currently a postdoctoral fellow studying genetic regulation in the bacterium Borrelia burgdorferi, which causes Lyme Disease. She holds a Ph.D. in Biomedical Sciences from the University of Toledo College of Medicine and Life Science in Ohio.

Somayeh Hooshmand is currently a postdoctoral fellow studying cholesterol metabolism and molecular, biochemical, and developmental processes that underlie genetic syndromes. She holds a Ph.D. in Human Genetics from University Putra Malaysia in Malaysia and a Ph.D. in Public Administration and Public Policy from Old Dominion University in Virginia.

Neysha Martinez-Orengo is currently a postdoctoral fellow studying the validation of candidate imaging biomarkers to better understand the pathophysiology of infectious diseases and improve non-invasive diagnostic techniques. She holds a Ph.D. in Biomedical Sciences from the Ponce Health Sciences University in Puerto Rico and is a member of the Puerto Rico-Science Policy Action Network (PR-SPAN).

Melissa Zajdel is currently a postdoctoral fellow studying how social network systems influence adaptation to chronic illnesses and stressful life events. She holds a Ph.D. in Psychology from Carnegie Mellon University and a M.S. in Global Health from Syracuse University.

\section{Acknowledgements}

The authors would like to acknowledge Dr. Federica La Russa for her help in preparing this manuscript. We are also thankful for the sponsored workshop series available to all authors interested in submitting to this special edition.

\section{Disclaimer}

This policy memo reflects the views of the authors and not necessarily those of their affiliations. The authors declare no conflicts of interest. 